\title{
Exact solution of the two-axis countertwisting Hamiltonian
}

\author{
Feng Pan ${ }^{1,3}$, Yao-Zhong Zhang ${ }^{2,4}$ *, and Jerry P. Draayer ${ }^{3}$ \\ ${ }^{1}$ Department of Physics, Liaoning Normal University, Dalian 116029, P. R. China \\ ${ }^{2}$ School of Mathematics and Physics, The University of Queensland, Brisbane, Qld 4072, Australia \\ ${ }^{3}$ Department of Physics and Astronomy, Louisiana State University, Baton Rouge, Louisiana 70803-4001, USA \\ ${ }^{4}$ CAS Key Laboratory of Theoretical Physics, Institute of Theoretical Physics \\ Chinese Academy of Sciences, Beijing 100190, P. R. China
}

(Dated: November 29, 2016)

\begin{abstract}
It is shown that the two-axis countertwisting Hamiltonian is exactly solvable when the quantum number of the total angular momentum of the system is an integer after the Jordan-Schwinger (differential) boson realization of the $\mathrm{SU}(2)$ algebra. Algebraic Bethe ansatz is used to get the exact solution with the help of the SU(1,1) algebraic structure, from which a set of Bethe ansatz equations of the problem is derived. It is shown that solutions of the Bethe ansatz equations can be obtained as zeros of the Heine-Stieltjes polynomials. The total number of the four sets of the zeros equals exactly to $2 J+1$ for a given integer angular momentum quantum number $J$, which proves the completeness of the solutions. It is also shown that double degeneracy in level energies may also occur in the $J \rightarrow \infty$ limit for integer $J$ case except a unique non-degenerate level with zero excitation energy.
\end{abstract}

PACS numbers: 42.50.Dv, 42.50.Lc, 32.60.+i

\section{Introduction}

Squeezed spin states of both Bose and Fermi many-body systems [1-8], where a component of the total angular momentum of an ensemble of spins has less uncertainty $[9,10]$ than other cases without quantum mechanical correlations, have been attracting great attention [11-14], not only because they are intrinsically interesting, but also because of being practically useful in precision measurements [2], quantum information, and fundamental tests of quantum mechanics [15]. As shown in [1], maximal squeezed spin states of a many-particle system can be generated by using the two-axis countertwisting mechanism, of which the Hamiltonian of the system is referred to as the two-axis countertwisting Hamiltonian. When the number of particles is small, the Hamiltonian can easily be diagonalized for a given quantum number of the total angular momentum of the system. However, one needs to handle a huge sparse matrix when the system contains an ensemble of a large number of particles [5$8,14]$. Exact analytical solution to the problem should be helpful, especially when one deals with a large number of particles. As noted in [16], up till now, there has been no general analytic solution available, though there were a few analytic treatments [1720] for a system with small number of particles.

In fact, significant progresses have been made in finding exact solutions of many-spin systems since the work of Bethe, Gaudin and Richardson [21-24]. Particularly, the Lipkin-Meshkov-Glick (LMG) model, which can be expressed in terms of the total angular momentum operators of the system up to their quadratic form, has been analytically solved by using the algebraic Bethe ansatz [25, 26]. The same problem can also be solved by using the Dyson boson realization of the SU(2) algebra [27-29], of which the solutions may be obtained from the Riccati differential equations [27, 28]. Discrete phase analysis of the model with applications to spin squeezing and entanglement was studied in [30]. In [31], it was shown that asymmetric rotor Hamiltonian can also be solved analytically by using the algebraic Bethe ansatz. However, though the two-axis countertwisting Hamiltonian is equivalent to a special case of the LMG model $[27,28]$ after an Euler rotation, the procedures used in $[25,26,31]$ can not be applied to the two-axis countertwisting Hamiltonian directly.

In this work, we show that the two-axis countertwisting Hamiltonian is indeed exactly solvable when the quantum number of the total angular momentum of the system is an integer after the Jordan-Schwinger (differential) boson realization of the SU(2) algebra. Similar to [31], exact solution to the problem will be derived based on the SU(1,1) algebraic structure after suitable transformations. Moreover, it is shown that solutions of the Bethe ansatz equations can be obtained from zeros of the Heine-Stieltjes polynomials, which, in turn, verifies the completeness of the solutions.

* Correspondence to: School of Mathematics and Physics, The University of Queensland, Brisbane, Qld 4072, Australia. Email address: yzz@maths.uq.edu.au. 


\section{The two-axis countertwisting Hamiltonian}

The two-axis countertwisting Hamiltonian may be written as [1]

$$
H_{\mathrm{TA}}=\frac{\chi}{2 i}\left(J_{+}^{2}-J_{-}^{2}\right),
$$

where $J_{ \pm}$are the angular momentum raising and lowering operators, $i=\sqrt{-1}$, and $\chi$ is a constant. The Hamiltoanian (1) is invariant under both parity and time reversal transformations, namely, it is $P T$-symmetric. Due to time-reversal symmetry, similar to the asymmetric rotor case [31], level energies of the system are all doubly degenerate when the quantum number of the total angular momentum is a half-integer. (1) is also equivalent to a special LMG Hamiltonian after rotation of the system by $\pi / 4$ around $z$ axis, of which the thermodynamic limit was studied in $[27,28]$ by using the Dyson boson (differential) realization and the corresponding Riccati differential equations.

Using the Jordan-Schwinger realization of SU(2), we have

$$
J_{+}=a^{\dagger} b, J_{-}=b^{\dagger} a, J_{0}=\frac{1}{2}\left(a^{\dagger} a-b^{\dagger} b\right),
$$

where $a, b$ and $a^{\dagger}, b^{\dagger}$ are boson annihilation and creation operators introduced. It can be observed that eigenstates of (1) may be expressed as

$$
|N, \zeta\rangle=F_{t}^{(\zeta)}\left(a^{\dagger 2}, b^{\dagger 2}\right)\left|v_{a}, v_{b}\right\rangle
$$

after the Jordan-Schwinger realization, where $F_{t}^{(\zeta)}\left(a^{\dagger 2}, b^{\dagger 2}\right)$ is a homogenous polynomial of degree $t$ with variables $\left\{a^{\dagger 2}, b^{\dagger 2}\right\}$, $\left|v_{a}, v_{b}\right\rangle$ is the boson pairing vacuum satisfying $a^{2}\left|v_{a}, v_{b}\right\rangle=0, b^{2}\left|v_{a}, v_{b}\right\rangle=0$, in which $\left|v_{a}, v_{b}\right\rangle=a^{\dagger v_{a}} b^{\dagger} v_{b}|0\rangle$ with the boson seniority numbers $v_{a}$ and $v_{b}=0$ or 1 , and $N=2 J=2 t+v_{a}+v_{b}$, in which $J$ is the quantum number of the total angular momentum of the system.

Then, we map the boson operators into the Bargmann variables with $a^{\dagger} \Leftrightarrow x, a \Leftrightarrow \partial / \partial x, b^{\dagger} \Leftrightarrow y, b \Leftrightarrow \partial / \partial y$. After this differential realization, however, there are two parts in the wavefunction. One is the collective part denoted as $F(x, y)$, which is a polynomial with even powers of $x$ and $y$. Another part denoted as $\Psi_{\text {in }}(x, y)$, which is a constant or proportional to $x$ or $y$. Thus, the total wavefunction in the Bargmann space may be expressed as $F(x, y) \Psi_{\text {in }}(x, y)$. As a consequence, the Hamiltonian (1) can be expressed as

$$
H_{\mathrm{TA}}=\frac{\chi}{2 i}\left(x^{2} \frac{\partial^{2}}{\partial y^{2}}-y^{2} \frac{\partial^{2}}{\partial x^{2}}+2 x^{2} \frac{\partial}{\partial y}\left(\frac{\partial}{\partial y}\right)_{\text {in }}-2 y^{2} \frac{\partial}{\partial x}\left(\frac{\partial}{\partial x}\right)_{\text {in }}\right),
$$

where $\left(\frac{\partial}{\partial y}\right)_{\text {in }}$ and $\left(\frac{\partial}{\partial y}\right)_{\text {in }}$ indicate that the derivatives are carried out for the intrinsic part $\Psi_{\text {in }}(x, y)$ only, while other derivatives are carried out for the collective part $F(x, y)$ only. Because the collective part $F(x, y)$ is a function of $x^{2}$ and $y^{2}$, we can make the following transformation:

$$
z_{1}=x^{2}, z_{2}=y^{2}
$$

We also have

$$
\frac{\partial}{\partial x}=2 x \frac{\partial}{\partial z_{1}}, \frac{\partial}{\partial y}=2 y \frac{\partial}{\partial z_{2}}
$$

and

$$
\frac{\partial^{2}}{\partial x^{2}}=2 \frac{\partial}{\partial z_{1}}+4 z_{1} \frac{\partial^{2}}{\partial z_{1}^{2}}, \frac{\partial^{2}}{\partial y^{2}}=2 \frac{\partial}{\partial z_{2}}+4 z_{2} \frac{\partial^{2}}{\partial z_{2}^{2}} .
$$

Substituting (5) - (7) into (4) and after separating the collective part from the intrinsic part, we get

$$
H_{\mathrm{TA}}=\frac{\chi}{i}\left(\left(1+2 \delta_{\hat{v}_{b} 1}\right) z_{1} \frac{\partial}{\partial z_{2}}-\left(1+2 \delta_{\hat{v}_{a}}\right) z_{2} \frac{\partial}{\partial z_{1}}+2 z_{1} z_{2}\left(\frac{\partial^{2}}{\partial z_{2}^{2}}-\frac{\partial^{2}}{\partial z_{1}^{2}}\right)\right),
$$


where $\hat{v}_{a}$ and $\hat{v}_{b}$ are seniority number operator of $a$ - and $b$-bosons, respectively. (8) is thus realized within the new twodimensional Bargmann space with variables $\left\{z_{1}, z_{2}\right\}$, while the intrinsic part characterized by the seniority numbers $v_{a}$ and $v_{b}$ only affects the first two terms in the Hamiltonian (8).

By mapping the Bargmann variables $\left\{z_{1}, z_{2}\right\}$ to new boson operators with $z_{1} \Leftrightarrow c^{\dagger}, \partial / \partial z_{1} \Leftrightarrow c, z_{2} \Leftrightarrow d^{\dagger}, \partial / \partial z_{2} \Leftrightarrow d$, Eq. (8) may be written as

$$
H_{\mathrm{TA}}=\frac{\chi}{i}\left(\left(1+2 \delta_{\hat{v}_{b} 1}\right) c^{\dagger} d-\left(1+2 \delta_{\hat{v}_{a} 1}\right) d^{\dagger} c+2 c^{\dagger} d^{\dagger}\left(d^{2}-c^{2}\right)\right) .
$$

The method outlined below works for integer $J$ case, but may not be applied to half-integer $J$ case directly. In fact, when $\hat{v}_{a}=\hat{v}_{b}=\hat{v}$, the Hamiltonian (9) can be expressed in terms of two canonical orthonormal boson modes

$$
a_{1}^{\dagger}=\sqrt{\frac{1}{2}}\left(c^{\dagger}+i d^{\dagger}\right), a_{2}^{\dagger}=\sqrt{\frac{1}{2}}\left(c^{\dagger}-i d^{\dagger}\right)
$$

with

$$
H_{\mathrm{TA}}=\chi\left(\left(1+2 \delta_{\hat{v} 1}\right)\left(a_{1}^{\dagger} a_{1}-a_{2}^{\dagger} a_{2}\right)+\left(a_{1}^{\dagger 2}-a_{2}^{\dagger 2}\right)\left(a_{1}^{2}+a_{2}^{2}\right)\right) .
$$

Though (11) is non-Hermitian, its eigenvalues are all real, mainly because of its equivalence to the original Hamiltonian (1) for this case. Since $v_{a}=v_{b}=v$, the total angular momentum of the system should be integer in this case with $J=0,1,2, \cdots$. It will be shown in the following that (11) can be solved analytically.

\section{Exact solution for integer $J$ cases}

In order to diagonalize (11), let us introduce two copies of SU(1,1) algebra generated by $\left\{S_{+}(1)=\frac{1}{2} a_{1}^{\dagger 2}, S_{-}(1)=\frac{1}{2} a_{1}^{2}\right.$, $\left.S_{0}(1)=\frac{1}{2}\left(a_{1}^{\dagger} a_{1}+\frac{1}{2}\right)\right\}$ and $\left\{S_{+}(2)=\frac{1}{2} a_{2}^{\dagger 2}, S_{-}(2)=\frac{1}{2} a_{2}^{2}, S_{0}(2)=\frac{1}{2}\left(a_{2}^{\dagger} a_{2}+\frac{1}{2}\right)\right\}$, which satisfy the following commutation relations:

$$
\left[S_{0}(l), S_{ \pm}(j)\right]=\delta_{l j} S_{ \pm}(j), \quad\left[S_{+}(l), S_{-}(j)\right]=-\delta_{l j} 2 S_{0}(j)
$$

Then, (11) can be written as

$$
H_{\mathrm{TA}}=2 \chi\left(\left(1+2 \delta_{\hat{v} 1}\right)\left(S_{0}(1)-S_{0}(2)\right)+2\left(S_{+}(1)-S_{+}(2)\right)\left(S_{-}(1)+S_{-}(2)\right)\right),
$$

which can be diagonalized under the Bethe ansatz

$$
\left|k, n_{1}, n_{2}, v ; \zeta\right\rangle=S_{+}\left(w_{1}^{(\zeta)}\right) S_{+}\left(w_{2}^{(\zeta)}\right) \cdots S_{+}\left(w_{k}^{(\zeta)}\right)\left|n_{1}, n_{2}, v\right\rangle
$$

with $J=2 k+n_{1}+n_{2}+v$, where $\left|n_{1}, n_{2}, v\right\rangle$ is the lowest weight state of the $\mathrm{SU}^{(l)}(1,1)$ for $l=1,2$, satisfying $S_{-}(l)\left|n_{1}, n_{2}, v\right\rangle=0$ and $2 S_{0}(l)\left|n_{1}, n_{2}, v\right\rangle=\left(n_{l}+\frac{1}{2}\right)\left|n_{1}, n_{2}, v\right\rangle$ with $n_{l}=0$ or 1 , and

$$
S_{+}(w)=\frac{1}{1-w} S_{+}(1)+\frac{1}{1+w} S_{+}(2) .
$$

By using the commutation relations (12), it can be proven that

$$
\left[S_{0}(1)-S_{0}(2), S_{+}(w)\right]=\frac{1}{1-w} S_{+}(1)-\frac{1}{1+w} S_{+}(2)=S_{+}+w S_{+}(w),
$$

where $S_{+}=S_{+}(1)-S_{+}(2)$

$$
\left[S_{-}(1)+S_{-}(2), S_{+}(w)\right]=\Lambda_{0}(w)=\frac{2 S_{0}(1)}{1-w}+\frac{2 S_{0}(2)}{1+w}
$$




$$
S_{+}\left(w_{1}, w_{2}\right)=\left[\left[S_{-}(1)+S_{-}(2), S_{+}\left(w_{1}\right)\right], S_{+}\left(w_{2}\right)\right]=\frac{2 w_{1}}{w_{1}-w_{2}} S_{+}\left(w_{1}\right)-\frac{2 w_{2}}{w_{1}-w_{2}} S_{+}\left(w_{2}\right)
$$

Using Eqs. (16) -(18), we can directly check that

$$
\begin{aligned}
\left(S_{0}(1)-S_{0}(2)\right)\left|k, n_{1}, n_{2}, v ; \zeta\right\rangle= & \left(S_{+}+w_{1}^{(\zeta)} S_{+}\left(w_{1}^{(\zeta)}\right)\right) S_{+}\left(w_{2}^{(\zeta)}\right) \cdots S_{+}\left(w_{k}^{(\zeta)}\right)\left|n_{1}, n_{2}, v\right\rangle \\
& +\cdots+S_{+}\left(w_{1}^{(\zeta)}\right) \cdots S_{+}\left(w_{k-1}^{(\zeta)}\right)\left(S_{+}+w_{k}^{(\zeta)} S_{+}\left(w_{k}^{(\zeta)}\right)\right)\left|n_{1}, n_{2}, v\right\rangle
\end{aligned}
$$

and

$$
\begin{aligned}
S_{+}\left(S_{-}(1)+S_{-}(2)\right)\left|k, n_{1}, n_{2}, v ; \zeta\right\rangle= & S_{+}\left(\left(\bar{\Lambda}_{0}\left(w_{1}^{(\zeta)}\right) S_{+}\left(w_{2}^{(\zeta)}\right) \cdots S_{+}\left(w_{k}^{(\zeta)}\right)\right.\right. \\
& \left.+\cdots+S_{+}\left(w_{1}^{(\zeta)}\right) \cdots S_{+}\left(w_{k-1}^{(\zeta)}\right) \bar{\Lambda}_{0}\left(w_{k}^{(\zeta)}\right)\right)\left|n_{1}, n_{2}, v\right\rangle \\
& +S_{+}\left(S_{+}\left(w_{1}^{(\zeta)}, w_{2}^{(\zeta)}\right) S_{+}\left(w_{3}^{(\zeta)}\right) \cdots S_{+}\left(w_{k}^{(\zeta)}\right)\right. \\
& +S_{+}\left(w_{1}^{(\zeta)}, w_{3}^{(\zeta)}\right) S_{+}\left(w_{2}^{(\zeta)}\right) S_{+}\left(w_{4}^{(\zeta)}\right) \cdots S_{+}\left(w_{k}^{(\zeta)}\right) \\
& +\cdots+S_{+}\left(w_{1}^{(\zeta)}, w_{k}^{(\zeta)}\right) S_{+}\left(w_{2}^{(\zeta)}\right) \cdots S_{+}\left(w_{k-1}^{(\zeta)}\right) \\
& +\cdots+S_{+}\left(w_{k}^{(\zeta)}, w_{1}^{(\zeta)}\right) S_{+}\left(w_{2}^{(\zeta)}\right) \cdots S_{+}\left(w_{k-1}^{(\zeta)}\right) \\
& +S_{+}\left(w_{k}^{(\zeta)}, w_{2}^{(\zeta)}\right) S_{+}\left(w_{3}^{(\zeta)}\right) \cdots S_{+}\left(w_{k-1}^{(\zeta)}\right) \\
& \left.+\cdots+S_{+}\left(w_{k}^{(\zeta)}, w_{k-1}^{(\zeta)}\right) S_{+}\left(w_{2}^{(\zeta)}\right) \cdots S_{+}\left(w_{k-2}^{(\zeta)}\right)\right)\left|n_{1}, n_{2}, v\right\rangle,
\end{aligned}
$$

where $\bar{\Lambda}_{0}(w)=\frac{n_{1}+\frac{1}{2}}{1-w}+\frac{n_{2}+\frac{1}{2}}{1+w}$.

Using Eqs. (18) -(20), one can prove that the eigen-equation $H_{\mathrm{TA}}\left|k, n_{1}, n_{2}, v ; \zeta\right\rangle=E_{k, n_{1}, n_{2}, v}^{(\zeta)}\left|k, n_{1}, n_{2}, v ; \zeta\right\rangle$ is fulfilled if and only if

$$
\frac{n_{1}+\frac{1}{2}}{1-w_{l}^{(\zeta)}}+\frac{n_{2}+\frac{1}{2}}{1+w_{l}^{(\zeta)}}+\frac{1}{2}\left(1+2 \delta_{v 1}\right)-\sum_{j \neq l} \frac{2 w_{j}^{(\zeta)}}{w_{l}^{(\zeta)}-w_{j}^{(\zeta)}}=0 \text { for } l=1,2, \cdots, k
$$

which are independent of the energy scale-factor $\chi$. The corresponding eigen-energy is given by

$$
E_{k, n_{1}, n_{2}, v}^{(\zeta)}=2 \chi\left(1+2 \delta_{v 1}\right)\left(\sum_{l=1}^{k} w_{l}^{(\zeta)}+\left(n_{1}-n_{2}\right) / 2\right)
$$

with $J=2 k+n_{1}+n_{2}+v$, where $k$ is the number of boson-quartets, $n_{1}$ and $n_{2}$ are the numbers of two different boson pairs, while $2 v$ is the total number of unpaired bosons, in which the bosons are the $a$ - and $b$-bosons introduced in (2). It can be inferred from (22) that the spectrum of the model after the Jordan-Schwinger two-boson realization is generated from the non-linear boson-quartet excitations based on the single-boson and the boson-pairing excitations, where the single-boson excitation affects both the scaling of the energy and the boson-quartet excitations, while the boson-pairing excitation energies contribute to the total energy linearly. Moreover, as shown previously [32-34], though the eigenstates provided in (14) are not normalized, they are always orthogonal with

$$
\left\langle k^{\prime}, n_{1}^{\prime}, 2 n_{2}^{\prime}, v^{\prime} ; \zeta^{\prime} \mid k, n_{1}, n_{2}, v ; \zeta\right\rangle=\left(\mathscr{N}\left(k, \zeta ; n_{1}, n_{2}, v\right)\right)^{-2} \delta_{k k^{\prime}} \delta_{n_{1} n_{1}^{\prime}} \delta_{n_{2} n_{2}^{\prime}} \delta_{v v^{\prime}} \delta_{\zeta \zeta^{\prime}}
$$

where $\mathscr{N}\left(k, \zeta ; n_{1}, n_{2}, v\right)$ is the corresponding normalization constant.

In order to find solutions of Eq. (21), one may change variables with $u_{l}=1 / w_{l}$. Then, Eq. (21) can be written as

$$
\frac{n_{1}+\frac{1}{2}}{u_{l}-1}+\frac{n_{2}+\frac{1}{2}}{u_{l}+1}+\frac{\frac{1}{2}\left(1+2 \delta_{v 1}\right)}{u_{l}}+\sum_{j \neq l} \frac{2}{u_{l}-u_{j}}=0 \text { for } l=1,2, \cdots, k
$$


According to the Heine-Stieltjes correspondence [32-34], zeros $\left\{u_{l}\right\}$ of the Heine-Stieltjes polynomials $y_{k}(u)$ of degree $k$ are roots of Eq. (24), where $y_{k}(u)$ should satisfy the following second-order Fuchsian equation:

$$
A(u) y_{k}^{\prime \prime}(u)+B(u) y_{k}^{\prime}(u)-V(u) y_{k}(u)=0 .
$$

Here, $A(u)=u\left(u^{2}-1\right)$, the polynomial $B(u)$ is given as

$$
\begin{gathered}
B(u) / A(u)=\frac{n_{1}+\frac{1}{2}}{u-1}+\frac{n_{2}+\frac{1}{2}}{u+1}+\frac{\frac{1}{2}\left(1+2 \delta_{v 1}\right)}{u}, \\
\frac{y_{k}^{\prime \prime}\left(u_{l}\right)}{y_{k}^{\prime}\left(u_{l}\right)}=\sum_{1 \leq j(\neq l) \leq k} \frac{2}{u_{l}-u_{j}},
\end{gathered}
$$

and $V(u)$ is a Van Vleck polynomial of degree 1, which is determined according to Eq. (25). Actually, the polynomial $y_{k}(u)$, of which the zeros satisfy (24), is an extended type of Niven or Lamé function, which is a special type of Heine-Stieltjes polynomials. Since $n_{1}+\frac{1}{2}, n_{2}+\frac{1}{2}$, and $\frac{1}{2}\left(1+2 \delta_{v 1}\right)$ are always real and positive, zeros of the Heine-Stieltjes polynomial are all real and satisfy the interlacing condition. Let these zeros be arranged as $u_{1}<u_{2}<\cdots<u_{k}$, which are in the union of two open intervals: $\left\{u_{1}, u_{2}, \cdots, u_{k}\right\} \in(-1,0) \cup(0,1)$. An electrostatic interpretation of the location of zeros of $y_{k}(u)$ may be stated as follows. Put three positive fixed fractional charges $\frac{1}{2} n_{2}+\frac{1}{4}, \frac{1}{4}\left(1+2 \delta_{v 1}\right)$, and $\frac{1}{2} n_{1}+\frac{1}{4}$ at $-1,0$, and +1 along a real line, respectively, and allow $k$ positive unit charges to move freely along the real line under such situation. There are $k+1$ different configurations for the position of these $k$ charges $\left\{u_{1}^{(\zeta)}, \cdots, u_{k}^{(\zeta)}\right\}$ with $\zeta=1,2, \cdots, k+1$, corresponding to global minimums of the total electrostatic energy of the system [32]. It follows from this that the total number of these configurations is exactly the number of ways to put the $k$ zeros into the two open intervals, which is $k+1$. Thus, there are $k+1$ different polynomials $y_{k}(u)$ for given $\left\{n_{1}, n_{2}, v\right\}$. Since $0 \leq n_{1}, n_{2}, v \leq 1$, for a given integer $J$, there are four different cases. Specifically, when $J$ is a fixed even integer, there are $k+1$ solutions with $J=2 k$ and $\left\{n_{1}=n_{2}=v=0\right\}$, while there are $k$ solutions for cases with $\left\{n_{1}=n_{2}=1, v=0\right\}$, or $\left\{n_{1}=v=1, n_{2}=0\right\}$, or $\left\{n_{2}=v=1, n_{1}=0\right\}$; when $J$ is a fixed odd integer, there are $k+1$ solutions when $J=2 k+1$ with $\left\{n_{1}=1, n_{2}=v=0\right\}$, or $\left\{n_{2}=1, n_{1}=v=0\right\}$, or $\left\{n_{1}=n_{2}=0, v=1\right\}$, while there are $k$ solutions for the case with $\left\{n_{1}=n_{2}=v=1\right\}$. It is obvious that the total number of different solutions equals exactly to $2 J+1$ for both even and odd $J$ cases, which proves the completeness of the solutions provided by (24) for the Hamiltonian (13). Therefore, for a given $J$, $2 J+1$ solutions in this Bethe ansatz approach split into 4 sets of solutions provided by (24) with different $\left\{n_{1}, n_{2}, v\right\}$.

Once the Bethe ansatz equations shown in (21) are solved, the eigenstate (14), up to a normalization constant, can be expressed in terms of the original $a$ - and $b$-boson operators as

$$
\left|k, n_{1}, n_{2}, v ; \zeta\right\rangle=\sum_{q=0}^{k} \sum_{\rho=0}^{k-q}\left(\begin{array}{c}
k-q \\
\rho
\end{array}\right)(-)^{\rho}(2 i)^{q} S_{q}^{(k, \zeta)} a^{\dagger 4 k-2 q-4 \rho} b^{\dagger 2 q+4 \rho}\left|n_{1}, n_{2}, v\right\rangle
$$

where

$$
S_{0}^{(k, \zeta)}=1, \quad S_{q \geq 1}^{(k, \zeta)}=\sum_{1 \leq \mu_{1} \neq \cdots \neq \mu_{q} \leq k} w_{\mu_{1}}^{(\zeta)} \cdots w_{\mu_{q}}^{(\zeta)}
$$

are the symmetric functions of $\left\{w_{1}^{(\zeta)}, \cdots, w_{k}^{(\zeta)}\right\}$, which are related to the expansion coefficients of $y_{k}(u)$ when it is expanded in terms of powers of $u$ [32-34]. Thus, when $J=2 k$, we have

$$
|J=2 k, \zeta\rangle=\left\{\begin{array}{cc}
\sum_{q=0}^{k} \sum_{\rho=0}^{k-q} \frac{(k-q) !(-)^{\rho}(2 i)^{q}}{(k-q-\rho) ! \rho !((4 k-2 q-4 \rho) !(2 q+4 \rho) !)^{-\frac{1}{2}}} S_{q}^{(k, \zeta)}|J=2 k, M=2 k-2 q-4 \rho\rangle & \text { for } n_{1}=n_{2}=v=0, \\
\sum_{q=0}^{k-1} \sum_{\rho=0}^{k-q-1} \frac{(k-q) !(-)^{\rho}}{(k-q-\rho) ! \rho !(2 i)^{-q}} S_{q}^{(k-1, \zeta)}\left(\frac{|J=2 k, M=2 k-2 q-4 \rho\rangle}{((4 k-2 q-4 \rho) !(2 q+4 \rho) !)^{-\frac{1}{2}}}+\frac{|J=2 k, M=2 k-2 q-4 \rho-4\rangle}{((4 k-2 q-4 \rho-4) !(2 q+4 \rho+4) !)^{-\frac{1}{2}}}\right) & \text { for } n_{1}=n_{2}=1, v=0, \\
\sum_{q=0}^{k-1} \sum_{\rho=0}^{k-q-1} \frac{(k-q) !(-)^{\rho}}{(k-q-\rho) ! \rho !(2 i)^{-q}} S_{q}^{(k-1, \zeta)}\left(\frac{|J=2 k, M=2 k-2 q-4 \rho-1\rangle}{((4 k-2 q-4 \rho-1) !(2 q+4 \rho+1) !)^{-\frac{1}{2}}}+\frac{i|J=2 k, M=2 k-2 q-4 \rho-3\rangle}{((4 k-2 q-4 \rho-3) !(2 q+4 \rho+3) !)^{-\frac{1}{2}}}\right) \text { for } n_{1}=v=1, n_{2}=0, \\
\sum_{q=0}^{k-1} \sum_{\rho=0}^{k-q-1} \frac{(k-q) !(-)^{\rho}}{(k-q-\rho) ! \rho !(2 i)^{-q}} S_{q}^{(k-1, \zeta)}\left(\frac{|J=2 k, M=2 k-2 q-4 \rho-1\rangle}{((4 k-2 q-4 \rho-1) !(2 q+4 \rho+1) !)^{-\frac{1}{2}}}-\frac{i|J=2 k, M=2 k-2 q-4 \rho-3\rangle}{((4 k-2 q-4 \rho-3) !(2 q+4 \rho+3) ! !)^{-\frac{1}{2}}}\right) \text { for } n_{2}=v=1, n_{1}=0 .
\end{array}\right.
$$


When $J=2 k+1$, we have

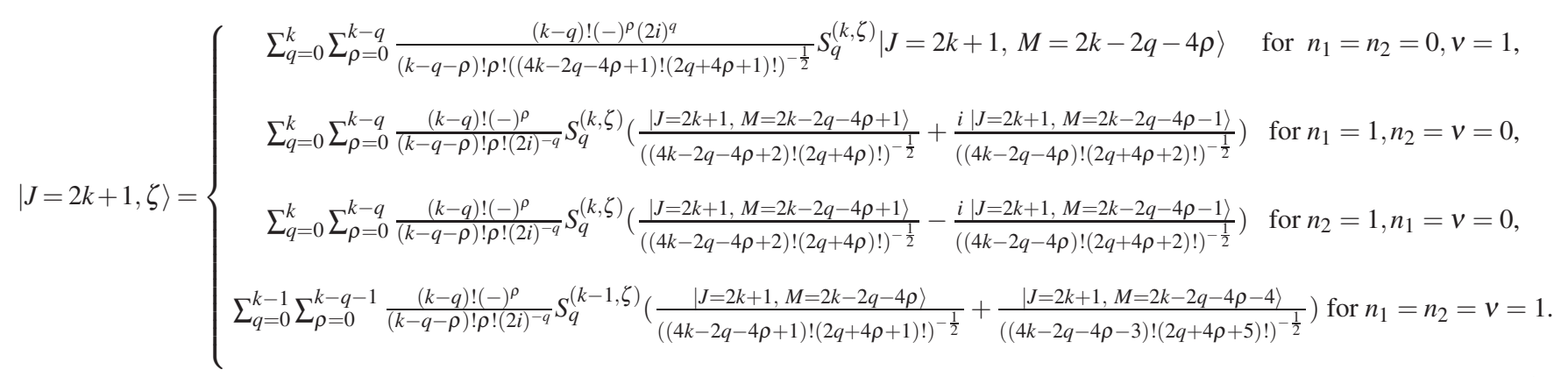

In order to solve (24) more easily, as shown in [32-35] for the extended Heine-Stieltjes polynomials, one may simply write

$$
y_{k}^{(\zeta)}(u)=\sum_{j=0}^{k} b_{j}^{(\zeta)} u^{j}
$$

where $\left\{b_{j}^{(\zeta)}\right\}(j=0,1, \cdots, k)$ are the $\zeta$-th set of the expansion coefficients to be determined. Substitution of (32) into (25) yields the condition to determine the corresponding Van Vleck polynomial with

$$
V^{(\zeta)}(u)=k\left(\frac{1}{2}\left(1+2 \delta_{v 1}\right)+n_{1}+n_{2}+k\right) u+g_{0}^{(\zeta)}
$$

The expansion coefficients $b_{j}^{(\zeta)}$ and $g_{0}^{(\zeta)}$ satisfy the following three-term relations:

$$
j\left(n_{1}-n_{2}\right) b_{j}^{(\zeta)}-(k-j+1)\left(k+j+n_{1}+n_{2}+\frac{1}{2}\left(1+2 \delta_{v 1}\right)-1\right) b_{j-1}^{(\zeta)}-(j+1)\left(\frac{1}{2}\left(1+2 \delta_{v 1}\right)+j\right) b_{j+1}=g_{0}^{(\zeta)} b_{j}^{(\zeta)}
$$

with $b_{j}^{(\zeta)}=0$ for $j \leq-1$ or $j \geq k+1$, which is equivalent to the eigenvalue problem with

$$
\mathbf{F b}^{(\zeta)}=g_{0}^{(\zeta)} \mathbf{b}^{(\zeta)}
$$

where the transpose of $\mathbf{b}^{(\zeta)}$ is related to the expansion coefficients $\left\{b_{j}^{(\zeta)}\right\}$ with $\left(\mathbf{b}^{(\zeta)}\right)^{\mathrm{T}}=\left(b_{0}^{(\zeta)}, b_{1}^{(\zeta)}, \cdots, b_{k-1}^{(\zeta)}, b_{k}^{(\zeta)}\right)$, and $\mathbf{F}$ is the $(k+1) \times(k+1)$ tridiagonal matrix with entries determined by $(34)$.

In addition, (32) can also be written in terms of the zeros $\left\{u_{j}^{(\zeta)}\right\}(j=1, \cdots, k)$ of $y_{k}^{(\zeta)}(u)$ with

$$
y_{k}^{(\zeta)}(u)=\prod_{j=1}^{k}\left(u-u_{j}^{(\zeta)}\right)=\sum_{q=0}^{k}(-1)^{q} \tilde{S}_{q}^{(k, \zeta)} u^{k-q},
$$

where $\tilde{S}_{q}^{(k, \zeta)}$ is the same symmetric function of $\left\{u_{1}^{(\zeta)}, \cdots, u_{k}^{(\zeta)}\right\}$ as that of $\left\{w_{1}^{(\zeta)}, \cdots, w_{k}^{(\zeta)}\right\}$ given in (29). In comparison of (36) with (32), we get

$$
b_{k-q}^{(\zeta)}=(-1)^{q} \tilde{S}_{q}^{(k, \zeta)}
$$

when the overall factor of $\left\{b_{j}^{(\zeta)}\right\}$ is chosen with $b_{k}^{(\zeta)}=1 \forall \zeta$. Hence, the symmetric function $\tilde{S}_{q}^{(k, \zeta)}$ is known after the expansion coefficients $\mathbf{b}^{(\zeta)}$ are obtained according to (35). Then, the symmetric functions $S_{q}^{(k, \zeta)}$ defined in (29) can be obtained from $\tilde{S}_{q}^{(k, \zeta)}$ with

$$
S_{q}^{(k, \zeta)}=\tilde{S}_{k-q}^{(k, \zeta)} \prod_{j=1}^{k} w_{j}^{(\zeta)}=(-1)^{q} b_{q}^{(\zeta)} / b_{0}^{(\zeta)}
$$


because $u_{j}^{(\zeta)}=1 / w_{j}^{(\zeta)}$, which can then be used in the eigenstates (30) and (31) to avoid unnecessary computation of $S_{q}^{(k, \zeta)}$ from $\left\{w_{1}^{(\zeta)}, \cdots, w_{k}^{(\zeta)}\right\}$

\section{Some numerical examples of the solution}

In order to demonstrate the method and solutions outlined previously, in this section, we provide some examples of the solution of (1) for integer $J$ cases. Similar to what was shown in [35], a Wolfram Mathematica package according to (32)-(35) is compiled, which is very efficient even when $J$ is a large number due to the fact that to generate and diagonalize a tridiagonal matrix are easier and more CPU time saving than other more complicated sparse matrices. When $J \leq 1$, the solutions are trivial with $k=0$, of which the eigen-energies are simply given by

$$
E_{k, n_{1}, n_{2}, v}^{(\zeta)}=\chi\left(1+2 \delta_{v 1}\right)\left(n_{1}-n_{2}\right)
$$

with $J=2 k+n_{1}+n_{2}+v$, while the corresponding eigenstates are given by (30) and (31) with $k=0$. When $J=2$, there is only one non-trivial case with $\left\{k=1, n_{1}=n_{2}=v=0\right\}$. When $J=3$, the only trivial case is that with $\left\{k=0, n_{1}=n_{2}=v=1\right\}$. When $J \geq 4$, all solutions are non-trivial.

The Heine-Stieltjes polynomials $y_{k}^{(\zeta)}(u)$ and the corresponding coefficient $g_{0}^{(\zeta)}$ in the Van Vleck polynomial shown in (33) up to $J=5$ are shown in Table I, while the $J=12$ case is provided in Table II. For any case, it can be verified that any zero of $y_{k}^{(\zeta)}(u)$ indeed lies in one of the intervals $(-1,0)$ and $(0,1)$. In addition, the Heine-Stieltjes polynomials $y_{k}^{(\zeta)}(u)$ is of order Int $[J / 2]$ or $\operatorname{Int}[J / 2+1]$, and always convergent when expanded in terms of $u$ in contrast to the characteristic polynomials of order $2 J+1$ generated from the original eigenvalue problem of (1), where Int $[z]$ is the integer part of $z$. By using (38), the eigen-energies given in (22) can also be expressed as

$$
E_{k, n_{1}, n_{2}, v}^{(\zeta)}=2 \chi\left(1+2 \delta_{v 1}\right)\left(-b_{1}^{(\zeta)} / b_{0}^{(\zeta)}+\left(n_{1}-n_{2}\right) / 2\right)
$$

with $J=2 k+n_{1}+n_{2}+v$, of which the corresponding numerical values are also provided in the last column of Tables I and II. It is shown in these Tables that there is a unique excited state with $E_{k, n_{1}, n_{2}, v}=0$ and $\left\{k=\operatorname{Int}[J / 2], n_{1}=n_{2}=v\right\}$ for $J \geq 4$, where $v=0$ when $J$ is even or $v=1$ when $J$ is odd. Except this unique state, there are many pairs of level energies close to each other, especially the lowest and the highest a few pairs, when $J$ is small. With the increasing of $J$, as shown in Table II for example, more pairs of levels seem almost degenerate. For example, the difference of excited energies of the ground and the first excited state $\left(E_{5.0,1,1}^{(1)}-E_{6,0,0.0}^{(1)}\right) / \chi$ is less than $10^{-6}$, though the numerical results up to the third decimal place shown in Table II are the same. The number of pairs of the almost double-degenerate levels increases with the increasing of $J$. It is expected that these pairs of level energies become the same when $J \rightarrow \infty$. The double degeneracy occurs is also due to time reversal symmetry of the system in the $J \rightarrow \infty$ limit, though it is not the case when $J$ is a finite integer. Since the double degeneracy always occurs for half-integer $J$ cases due to time reversal symmetry, it can be inferred that the double degeneracy should also occur for integer $J$ cases after removing the unique level with excitation energy being zero when $J \rightarrow \infty$ because there will be no difference of integer $J$ cases from half-integer $J$ cases in the $J \rightarrow \infty$ limit except the unique state in the integer $J$ case. Furthermore, with the increasing of $J$, the level energy distribution of pairs of the almost double-degenerate levels is symmetric with respect to $E=0$, which is the excitation energy of the unique state, namely, there are $\operatorname{Int}[J / 2]$ almost doubly degenerate levels with energies $E_{r}>0$ and the same number of pairs of almost doubly degenerate levels with energies $-E_{r}<0$ for $r=1,2, \cdots$, Int $[J / 2]$, which should be helpful in evaluating the time evolution matrix [36] of the system in the large $J$ limit. Anyway, once the expansion coefficients $\mathbf{b}$ are obtained, the results can be used for constructing eigenstates according to (30) and (31), which can then be used to calculate and analyze physical quantities in the system.

\section{SUMMARY}

In this work, by using the Jordan-Schwinger (differential) boson realization of the SU(2) algebra, it is shown that the two-axis countertwisting Hamiltonian is exactly solvable with the help of the algebraic Bethe ansatz when the quantum number of the total angular momentum of the system $J$ is an integer. Here, exactly or analytically solvable Hamiltonian means that its entire spectral problem can be reduced to an algebraic one, which is also related to its integrability [37]. Its solutions can then be obtained algebraically with eigenvalues expressed in terms of roots of a set of Bethe ansatz equations. Though the Hamiltonian for half-integer $J$ case seems also solvable, the procedure shown in this work can not be applied to half-integer $J$ case directly, 
TABLE I: The Heine-Stieltjes Polynomials $y_{k}^{(\zeta)}(u), g_{0}^{(\zeta)}$ of the corresponding Van Vleck Polynomial $V^{(\zeta)}(u)$, and the corresponding eigenen$\operatorname{ergy} E_{k, n_{1}, n_{2}, v}^{(\zeta)} / \chi$ of the Hamiltonian (1) for $J \leq 5$, where the order of $\zeta$ is arranged according to the value of the eigen-energy of (1) for a given set of $\left\{k, n_{1}, n_{2}, v\right\}$.

\begin{tabular}{|c|c|c|c|c|}
\hline \multicolumn{2}{|c|}{$J\left\{k, \zeta ; n_{1}, n_{2}, v\right\}$} & \multirow{2}{*}{$\frac{y_{k}^{(\zeta)}(u)}{1}$} & \multirow{2}{*}{$\begin{array}{c}g_{0}^{(\zeta)} \\
0\end{array}$} & \multirow{2}{*}{$\begin{array}{c}E_{k, n_{1}, n_{2}, v}^{(\zeta)} / \chi \\
0\end{array}$} \\
\hline$\overline{0}$ & $\{0,1 ; 0,0,0\}$ & & & \\
\hline \multirow[t]{3}{*}{1} & $\{0,1 ; 1,0,0\}$ & 1 & 0 & 1 \\
\hline & $\{0,1 ; 0,0,1\}$ & 1 & 0 & 0 \\
\hline & $\{0,1 ; 0,1,0\}$ & 1 & 0 & -1 \\
\hline \multirow[t]{5}{*}{2} & $\{0,1 ; 1,0,1\}$ & 1 & 0 & 3 \\
\hline & $\{0,1 ; 1,1,0\}$ & 1 & 0 & 0 \\
\hline & $\{0,1 ; 0,1,1\}$ & 1 & 0 & -3 \\
\hline & $\{1,1 ; 0,0,0\}$ & $0.57735+u$ & -0.866025 & -3.4641 \\
\hline & $\{1,2 ; 0,0,0\}$ & $-0.57735+u$ & 0.866025 & 3.4641 \\
\hline \multirow[t]{7}{*}{3} & $\{0,1 ; 1,1,1\}$ & 1 & 0 & 0 \\
\hline & $\{1,1 ; 0,0,1\}$ & $0.774597+u$ & -1.93649 & -7.74597 \\
\hline & $\{1,2 ; 0,0,1\}$ & $-0.774597+u$ & 1.93649 & 7.74597 \\
\hline & $\{1,1 ; 0,1,0\}$ & $0.289898+u$ & -1.72474 & -7.89898 \\
\hline & $\{1,2 ; 0,1,0\}$ & $-0.689898+u$ & 0.724745 & 1.89898 \\
\hline & $\{1,1 ; 1,0,0\}$ & $0.689898+u$ & -0.724745 & -1.89898 \\
\hline & $\{1,2 ; 1,0,0\}$ & $-0.289898+u$ & 1.72474 & 7.89898 \\
\hline \multirow[t]{9}{*}{4} & $\{2,1 ; 0,0,0\}$ & $0.142857+1.03016 u+u^{2}$ & 1.03016 & -14.4222 \\
\hline & $\{2,2 ; 0,0,0\}$ & $-0.6+u^{2}$ & 0 & 0 \\
\hline & $\{2,3 ; 0,0,0\}$ & $0.142857-1.03016 u+u^{2}$ & -1.03016 & 14.4222 \\
\hline & $\{1,1 ; 0,1,1\}$ & $0.527202+u$ & -2.84521 & -14.3808 \\
\hline & $\{1,2 ; 0,1,1\}$ & $-0.812917+u$ & 1.84521 & 4.38083 \\
\hline & $\{1,1 ; 1,0,1\}$ & $0.812917+u$ & -1.84521 & -4.38083 \\
\hline & $\{1,2 ; 1,0,1\}$ & $-0.527202+u$ & 2.84521 & 14.3808 \\
\hline & $\{1,1 ; 1,1,0\}$ & $0.377964+u$ & -1.32288 & -5.2915 \\
\hline & $\{1,2 ; 1,1,0\}$ & $-0.377964+u$ & 1.32288 & 5.2915 \\
\hline \multirow[t]{11}{*}{5} & $\{2,1 ; 0,0,1\}$ & $0.333333+1.27657 u+u^{2}$ & 1.27657 & -22.9783 \\
\hline & $\{2,2 ; 0,0,1\}$ & $-0.714286+u^{2}$ & 0 & 0 \\
\hline & $\{2,3 ; 0,0,1\}$ & $0.333333-1.27657 u+u^{2}$ & -1.27657 & 22.9783 \\
\hline & $\{2,1 ; 0,1,0\}$ & $0.0706856+0.777127 u+u^{2}$ & 0.777127 & -22.9883 \\
\hline & $\{2,2 ; 0,1,0\}$ & $-0.40046-0.347913 u+u^{2}$ & -0.347913 & -2.73757 \\
\hline & $\{2,3 ; 0,1,0\}$ & $0.186917-1.09588 u+u^{2}$ & -1.09588 & 10.7259 \\
\hline & $\{2,1 ; 1,0,0\}$ & $0.186917+1.09588 u+u^{2}$ & 1.09588 & -10.7259 \\
\hline & $\{2,2 ; 1,0,0\}$ & $-0.40046+0.347913 u+u^{2}$ & 0.347913 & 2.73757 \\
\hline & $\{2,3 ; 1,0,0\}$ & $0.0706856-0.777127 u+u^{2}$ & -0.777127 & 22.9883 \\
\hline & $\{1,1 ; 1,1,1\}$ & $0.57735+u$ & -2.59808 & -10.3923 \\
\hline & $\{1,2 ; 1,1,1\}$ & $-0.57735+u$ & 2.59808 & 10.3923 \\
\hline
\end{tabular}

which, therefore, has not been addressed in the present study. It is shown that solutions of the Bethe ansatz equations can be obtained as zeros of the Heine-Stieltjes polynomials determined by the second order Fuchsian type differential equation. It is verified that the inverse of the zeros are all real and within the two open intervals $(-1,0)$ and $(0,1)$. The total number of the four sets of the zeros equals exactly to $2 J+1$ for a given $J$, which proves the completeness of the solutions. It is also observed that the matrix in determining the zeros is also tridiagonal and $\operatorname{Int}[J / 2]$ or $\operatorname{Int}[J / 2+1]$ dimensional. Moreover, there is a non-degenerate unique level with the excitation energy being zero. It is revealed that there are many pairs of level energies, especially the first a few lowest and the last a few highest levels, being almost double-degenerate. The number of the almost double-degenerate levels increases with the increasing of $J$. Since the double degeneracy always occurs in half-integer $J$ case due to time reversal symmetry, it can be inferred that the double degeneracy should also occur in integer $J$ case when $J \rightarrow \infty$ except the unique level. 
TABLE II: The same as Table I, but for $J=12$.

\begin{tabular}{|c|c|c|c|}
\hline$\left\{k, \zeta ; n_{1}, n_{2}, v\right\}$ & $\underline{y_{k}^{(\zeta)}(u)}$ & $g_{0}^{(\bar{\zeta})}$ & $E_{k, n_{1}, n_{2}, v}^{(\zeta)} / \chi$ \\
\hline$\{6,1 ; 0,0,0\}$ & $0.000541694+0.0376684 u+0.429523 u^{2}+1.80288 u^{3}+3.43433 u^{4}+3.0234 u^{5}+u^{6}$ & 0.0376684 & -139.076 \\
\hline$\{6,2 ; 0,0,0\}$ & $-0.00319288-0.123789 u-0.758387 u^{2}-1.34125 u^{3}-0.0153776 u^{4}+1.68567 u^{5}+u^{6}$ & -0.123789 & -77.5408 \\
\hline$\{6,3 ; 0,0,0\}$ & $0.0165927+0.24233 u+0.37415 u^{2}-0.847361 u^{3}-1.35063 u^{4}+0.634983 u^{5}+u^{6}$ & 0.24233 & -29.2092 \\
\hline$\{6,4 ; 0,0,0\}$ & $-0.0497738+0.647059 u^{2}-1.57143 u^{4}+u^{6}$ & 0 & 0 \\
\hline$\{6,5 ; 0,0,0\}$ & $0.0165927-0.24233 u+0.37415 u^{2}+0.847361 u^{3}-1.35063 u^{4}-0.634983 u^{5}+u^{6}$ & -0.24233 & 29.2092 \\
\hline$\{6,6 ; 0,0,0\}$ & $-0.00319288+0.123789 u-0.758387 u^{2}+1.34125 u^{3}-0.0153776 u^{4}-1.68567 u^{5}+u^{6}$ & 0.123789 & 77.5408 \\
\hline$\{6,7 ; 0,0,0\}$ & $0.000541694-0.0376684 u+0.429523 u^{2}-1.80288 u^{3}+3.43433 u^{4}-3.0234 u^{5}+u^{6}$ & -0.0376684 & 139.076 \\
\hline$\{5,1 ; 0,1,1\}$ & $0.00637508+0.144583 u+0.906987 u^{2}+2.29763 u^{3}+2.5234 u^{4}+u^{5}$ & 0.144583 & -139.076 \\
\hline$\{5,2 ; 0,1,1\}$ & $-0.027919-0.346847 u-1.01394 u^{2}-0.483239 u^{3}+1.18565 u^{4}+u^{5}$ & -0.346847 & -77.5399 \\
\hline$\{5,3 ; 0,1,1\}$ & $0.0854689+0.368501 u-0.238076 u^{2}-1.29453 u^{3}+0.127591 u^{4}+u^{5}$ & 0.368501 & -28.8692 \\
\hline$\{5,4 ; 0,1,1\}$ & $-0.114417+0.232435 u+0.669984 u^{2}-1.0747 u^{3}-0.699757 u^{4}+u^{5}$ & 0.232435 & 9.18883 \\
\hline$\{5,5 ; 0,1,1\}$ & $0.0520016-0.472687 u+0.993523 u^{2}+0.0512233 u^{3}-1.62042 u^{4}+u^{5}$ & -0.472687 & 51.5391 \\
\hline$\{5,6 ; 0,1,1\}$ & $-0.0137782+0.252043 u-1.33025 u^{2}+2.91356 u^{3}-2.82082 u^{4}+u^{5}$ & 0.252043 & 106.758 \\
\hline$\{5,1 ; 1,0,1\}$ & $0.0137782+0.252043 u+1.33025 u^{2}+2.91356 u^{3}+2.82082 u^{4}+u^{5}$ & 0.252043 & -106.758 \\
\hline$\{5,2 ; 1,0,1\}$ & $-0.0520016-0.472687 u-0.993523 u^{2}+0.0512233 u^{3}+1.62042 u^{4}+u^{5}$ & -0.472687 & -51.5391 \\
\hline$\{5,3 ; 1,0,1\}$ & $0.114417+0.232435 u-0.669984 u^{2}-1.0747 u^{3}+0.699757 u^{4}+u^{5}$ & 0.232435 & -9.18883 \\
\hline$\{5,4 ; 1,0,1\}$ & $-0.0854689+0.368501 u+0.238076 u^{2}-1.29453 u^{3}-0.127591 u^{4}+u^{5}$ & 0.368501 & 28.8692 \\
\hline$\{5,5 ; 1,0,1\}$ & $0.027919-0.346847 u+1.01394 u^{2}-0.483239 u^{3}-1.18565 u^{4}+u^{5}$ & -0.346847 & 77.5399 \\
\hline$\{5,6 ; 1,0,1\}$ & $-0.00637508+0.144583 u-0.906987 u^{2}+2.29763 u^{3}-2.5234 u^{4}+u^{5}$ & 0.144583 & 139.076 \\
\hline$\{5,1 ; 1,1,0\}$ & $0.00133825+0.0714339 u+0.618781 u^{2}+1.87815 u^{3}+2.32082 u^{4}+u^{5}$ & 0.0714339 & -106.758 \\
\hline$\{5,2 ; 1,1,0\}$ & $-0.00736718-0.189934 u-0.724024 u^{2}-0.383373 u^{3}+1.12091 u^{4}+u^{5}$ & -0.189934 & -51.5621 \\
\hline$\{5,3 ; 1,1,0\}$ & $0.0351315+0.207975 u-0.233945 u^{2}-1.03515 u^{3}+0.257387 u^{4}+u^{5}$ & 0.207975 & -11.8398 \\
\hline$\{5,4 ; 1,1,0\}$ & $-0.0351315+0.207975 u+0.233945 u^{2}-1.03515 u^{3}-0.257387 u^{4}+u^{5}$ & 0.207975 & 11.8398 \\
\hline$\{5,5 ; 1,1,0\}$ & $0.00736718-0.189934 u+0.724024 u^{2}-0.383373 u^{3}-1.12091 u^{4}+u^{5}$ & -0.189934 & 51.5621 \\
\hline$\{5,6 ; 1,1,0\}$ & $-0.00133825+0.0714339 u-0.618781 u^{2}+1.87815 u^{3}-2.32082 u^{4}+u^{5}$ & 0.0714339 & 106.758 \\
\hline
\end{tabular}

The level energy distribution of the almost double-degenerate levels is symmetric with respect to the unique level, which should be helpful in evaluating the time evolution matrix [36] of the system in the large $J$ limit. The procedure outlined may be helpful in calculating physical quantities in the system in order to produce maximal squeezed spin states of many-particle systems.

Support from the U. S. National Science Foundation (OCI-0904874, ACI -1516338), U.S. Department of Energy (DESC0005248), the Southeastern Universities Research Association, the China-U. S. Theory Institute for Physics with Exotic Nuclei (CUSTIPEN) (DE-SC0009971), the National Natural Science Foundation of China (11375080, and 11675071), the Australian Research Council Discovery Project DP140101492, and the LSU-LNNU joint research program (9961) is acknowledged.

[1] M. Kitagawa and M. Ueda, Phys. Rev. A 47, 5138 (1993).

[2] D. J. Wineland, J. J. Bollinger, W. M. Itano, F. L. Moore, and D. J. Heinzen, Phys. Rev. A 46, R6797 (1992).

[3] D. J. Wineland, J. J. Bollinger, W. M. Itano, and D. J. Heinzen, Phys. Rev. A 50, R67 (1994).

[4] A. Sørensen and K. Mømer, Phys. Rev. Lett. 86, 4431 (2001).

[5] J. Hald, J. L. Sørensen, C. Schori, and E. S. Polzik, Phys. Rev. Lett. 83, 1319 (1999).

[6] I. D. Leroux, M. H. Schleier-Smith, and V. Vuletć, Phys. Rev. Lett. 104, 073602 (2010).

[7] C. D. Hamley, C. S. Gerving, T. M. Hoang, E. M. Bookjans and M. S. Chapman, Nature Phys. 8, 305 (2012).

[8] H. Strobel, W. Muessel, D. Linnemann, T. Zibold, Science 345, 424 (2014).

[9] A. Kuzmich, L. Mandel, and N. P. Bigelow, Phys. Rev. Lett. 85, 1594 (2000).

[10] T. Takano, M. Fuyama, R. Namiki, and Y. Takahashi, Phys. Rev. Lett. 102, 033601 (2009).

[11] V. Meyer, M. A. Rowe, D. Kielpinski, C. A. Sackett, W. M. Itano, C. Monroe, and D. J. Wineland, Phys. Rev. Lett. 86, 5870 (2001).

[12] J. Estéve, C. Gross, A. Weller, S. Giovanazzi, and M. K. Oberthaler, Nature 455, 1216 (2008).

[13] J. Appel, P. J. Windpassinger, D. Oblak, U. B. Hoff, N. Kjægaard, and E. S. Polzik, PNAS 106, 10960 (2009).

[14] M. H. Schleier-Smith, I. D. Leroux, and V. Vuletć, Phys. Rev. Lett. 104, 073604 (2010). 
[15] S. L. Braunstein, and P. van Loock, Rev. Mod. Phys. 77, 513 (2005).

[16] M. Bhattacharya, Analytical solvability of the two-axis countertwisting spin squeezing Hamiltonian (2015), arXiv:1509.08530.

[17] P. K. Pathak, R. N. Deb, N. Nayak and B. Dutta-Roy, J. Phys. A 41, 145302 (2008).

[18] M. Jafarpour and A. Akhound, Phys. Lett. A 372, 2374 (2008).

[19] I. Stewart, Galois Theory (Chapman and Hall, United Kingdom, 2000).

[20] J. Ma, X. Wang, C. P. Sun and F. Nori, Phys. Rep. 509, 89 (2011).

[21] H. Bethe, Z. Phys. 71, 205 (1931).

[22] M. Gaudin, J. Phys. (Paris) 37, 1087 (1976).

[23] R. W. Richardson, Phys. Lett. 3, 277 (1963); J. Math. Phys. 6, 1034 (1965).

[24] R. W. Richardson and N. Sherman, Nucl. Phys. 52, 221 (1964).

[25] F. Pan and J. P. Draayer, Phys. Lett. B 451, 1 (1999).

[26] H. Morita, H. Ohnishi, J. da Providêcia, S. Nishiyama, Nucl. Phys. B, 737, 337 (2006).

[27] P. Ribeiro, J. Vidal, and R. Mosseri, Phys. Rev. Lett. 99, 050402 (2007).

[28] P. Ribeiro, J. Vidal, and R. Mosseri, Phys. Rev. E 78, 021106 (2008).

[29] Y.-H Lee, J. Links, and Y.-Z. Zhang, Nonlinearity 24, 1975 (2011).

[30] M. A. Marchiolli, D. Galetti, T. Debarba, Int. J. Quant. Info. 11, 1330001 (2013).

[31] F. Pan and J. P. Draayer, Ann. Phys. (N. Y.) 275, 224 (1999).

[32] F. Pan, L. Bao, L. Zhai, X. Cui, and J. P. Draayer, J. Phys. A: Math. Theor. 44, 395305 (2011).

[33] X. Guan, K. D. Launey, M. Xie, L. Bao, F. Pan, and J. P. Draayer, Phys. Rev. C 86, 024313 (2012).

[34] F. Pan, B. Li, Y.-Z. Zhang, and J. P. Draayer, Phys. Rev. C 88, 034305 (2013).

[35] X. Guan, K. D. Launey, M. Xie, L. Bao, F. Pan, J. P. Draayer, Comp. Phys. Commun. 185, 2714 (2014).

[36] F. De Zela, Symmetry 6, 329 (2014).

[37] G. Ortiz. and R. Somma, J. Dukelsky, and S. Rombouts, Nucl. Phys. B 707, 421 (2005). 